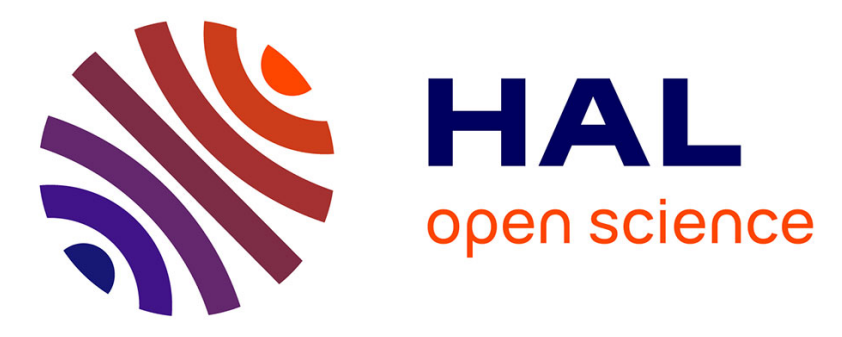

\title{
Characterizing Thermal Mixing Dynamic Nuclear Polarization via Cross-Talk between Spin Reservoirs
}

David Guarin, Sina Marhabaie, Alberto Rosso, Daniel Abergel, Geoffrey

Bodenhausen, Konstantin L. Ivanov, Dennis Kurzbach

\section{- To cite this version:}

David Guarin, Sina Marhabaie, Alberto Rosso, Daniel Abergel, Geoffrey Bodenhausen, et al.. Characterizing Thermal Mixing Dynamic Nuclear Polarization via Cross-Talk between Spin Reservoirs. Journal of Physical Chemistry Letters, 2017, 8 (22), pp.5531-5536. 10.1021/acs.jpclett.7b02233 . hal-02017641

\section{HAL Id: hal-02017641 \\ https://hal.sorbonne-universite.fr/hal-02017641}

Submitted on 13 Feb 2019

HAL is a multi-disciplinary open access archive for the deposit and dissemination of scientific research documents, whether they are published or not. The documents may come from teaching and research institutions in France or abroad, or from public or private research centers.
L'archive ouverte pluridisciplinaire HAL, est destinée au dépôt et à la diffusion de documents scientifiques de niveau recherche, publiés ou non, émanant des établissements d'enseignement et de recherche français ou étrangers, des laboratoires publics ou privés. 


\title{
Characterizing Thermal Mixing Dynamic Nuclear Polarization via ${ }_{2}$ Cross-Talk between Spin Reservoirs
}

\author{
3 David Guarin, ${ }^{\dagger, \dagger}$ Sina Marhabaie, ${ }^{\dagger, \dagger}$ Alberto Rosso, ${ }^{\dagger, \S}$ Daniel Abergel, ${ }^{\dagger, \dagger}$ Geoffrey Bodenhausen, ${ }^{\dagger, \ddagger}$ \\ ${ }_{4}$ Konstantin L. Ivanov, ${ }^{*}, \|, \perp$ and Dennis Kurzbach $*,+, \odot$ \\ $5{ }^{\dagger}$ Département de Chimie, Ecole Normale Supérieure, PSL Research University, UPMC Univ Paris 06, CNRS, Laboratoire des \\ 6 Biomolécules (LBM), 24 rue Lhomond, 75005 Paris, France \\ 7 \$Sorbonne Universités, UPMC Univ Paris 06, Ecole Normale Supérieure, CNRS, Laboratoire des Biomolécules (LBM), 75005 Paris, \\ 8 France \\ $9{ }^{\S}$ Laboratoire Physique Théorique et Modèles Statistiques (LPTMS), Université Paris-Sud, Université Paris-Saclay, CNRS, 91405 \\ 10 Orsay, France \\ 11 "International Tomography Center SB RAS, Institutskaya 3a, Novosibirsk 630090, Russia \\ $12{ }^{\perp}$ Novosibirsk State University, Pirogova 2, Novosibirsk 630090, Russia
}

13 ABSTRACT: Dynamic nuclear polarization (DNP) embraces

14 a family of methods to increase signal intensities in nuclear

15 magnetic resonance (NMR) spectroscopy. Despite extensive

16 theoretical work that allows one to distinguish at least five

17 distinct mechanisms, it remains challenging to determine the

18 relative weights of the processes that are responsible for DNP

19 in state-of-the-art experiments operating with stable organic

20 radicals like nitroxides at high magnetic fields and low temperatures. Specifically, determining experimental conditions where DNP involves thermal mixing, which denotes a spontaneous heat exchange between different spin reservoirs, remains challenging. We propose an experimental approach to ascertain the prevalence of the thermal mixing regime by monitoring characteristic signature properties of the time evolution of the hyperpolarization. We find that thermal mixing is the dominant DNP mechanism at high nitroxide radical concentrations, while a mixture of different mechanisms prevails at lower concentrations.
${ }_{28}^{27} \bigcirc$ ynamic nuclear polarization (DNP) is a powerful method for enhancing the polarization of nuclear spins with the 29 aim of boosting weak nuclear magnetic resonance (NMR) 30 signals. In recent years, DNP has experienced a remarkable 31 "renaissance" due to novel methodological developments, in 32 particular for solid-state DNP (MAS-DNP) $)^{1-4}$ and dissolution 33 DNP (D-DNP), ${ }^{5-10}$ which enables many new applications in 34 practically all fields of $\mathrm{NMR}^{10,11}$ and $\mathrm{MRI}^{5,7}$ ranging from in35 cell metabolomics to cancer monitoring in humans. At the heart 36 of DNP lies the transfer of electron spin polarization to nuclear 37 spins upon pumping electron paramagnetic resonance (EPR) 38 transitions of stable mono- or biradicals. To widen the range of 39 applications of DNP and to optimize the enhancements, a 40 thorough understanding of the underlying mechanisms is a 41 prerequisite. At this time, five distinct DNP mechanisms are 42 known to play a role in solids: ${ }^{12-14}$ the solid effect (SE), the 43 differential solid effect (DSE), the cross-effect (CE), thermal 44 mixing (TM) and the Overhauser effect (OE). While the latter 45 exploits dissipative cross-relaxation, the former four mecha46 nisms make use of coherent polarization transfer. The SE is due 47 to pumping "forbidden" combinations of EPR and NMR 48 transitions; since it involves an isolated electron spin and an 49 isolated nuclear spin, this mechanism is most efficient when using dilute monoradicals. A more efficient DNP mechanism 50 that dominates for dilute biradicals or concentrated mono- 51 radicals is provided by the $\mathrm{CE}$, which requires a pair of electron 52 spins so that "triple spin flips" (of two electron spins, $S S^{\text {', and } 53}$ one nuclear spin) can occur (denoted $S S^{\prime} I$ for $I={ }^{1} \mathrm{H}$, or $S S^{\prime} I^{\prime} 54$ for other nuclei $I^{\prime}$ such as ${ }^{2} \mathrm{H},{ }^{13} \mathrm{C},{ }^{31} \mathrm{P}$, etc.) Finally, 55 concentrated monoradicals with strong intermolecular inter- 56 actions can lead to TM, a mechanism for which thermodynamic 57 concepts can be used ${ }^{15-17}$ to describe polarization transfer in 58 terms of heat exchange between different reservoirs. TM is 59 effective only when the EPR spectral width $\Delta \nu_{e}$, given either by 60 homogeneous line broadening or electronic spectral diffusion 61 (eSD), exceeds the nuclear Zeeman interaction frequency 62 $\nu_{\mathrm{I}}{ }^{18,19}$

Various definitions for TM have been given. ${ }^{14-17}$ Here we 64 consider that TM entails three important properties. (1) 65 Electron spins, even under microwave irradiation, display a 66 behavior that can be described using thermodynamic concepts. 67 Namely, their description involves two heat reservoirs: a 68 
a)
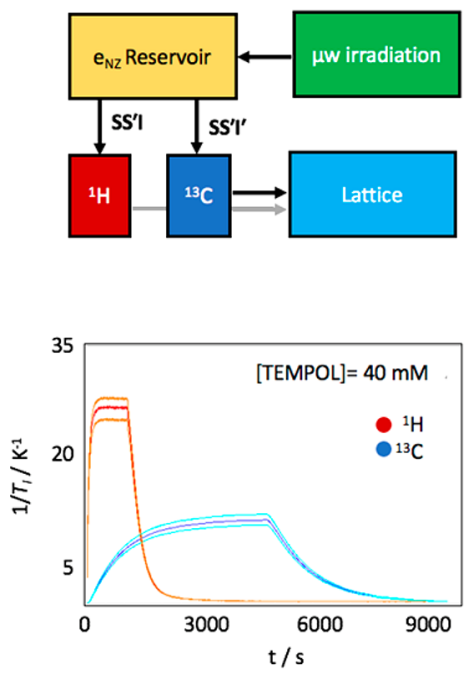

b)
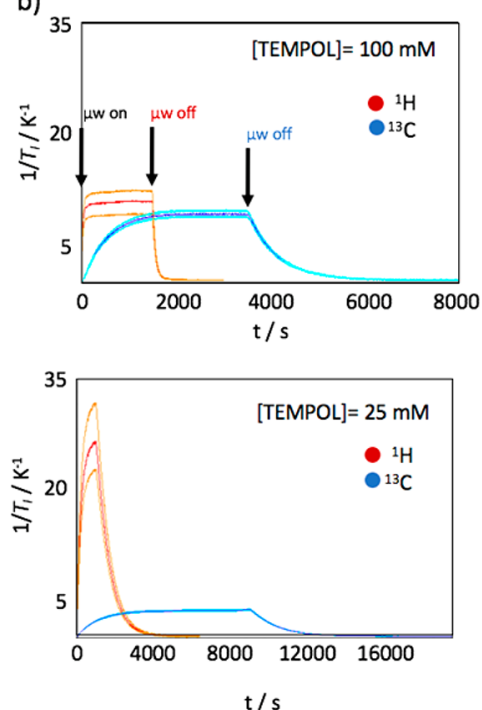
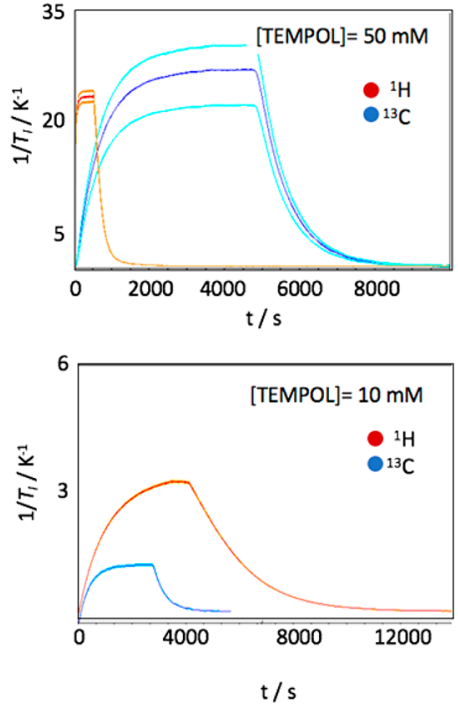

Figure 1. (a) Energy reservoirs involved in experiment $\mathbf{A}$ that monitors the buildup of the polarizations $P\left(I={ }^{1} \mathrm{H}\right)$ and $P\left(I^{\prime}={ }^{13} \mathrm{C}\right)$ after switching on the saturation of the EPR spectrum by microwaves $(\mu \mathrm{w})$, and their decay after interruption of the $\mu \mathrm{w}$ irradiation. The $\mu \mathrm{w}$ irradiation cools down the non-Zeeman dipolar electron reservoir $\mathrm{e}_{\mathrm{NZ}}$; as a result, the ${ }^{1} \mathrm{H}$ and ${ }^{13} \mathrm{C}$ spins are polarized simultaneously via heat exchange between the reservoirs. Nuclear relaxation proceeds via dissipation of energy to the lattice. (b) DNP build-up curves and decay of polarizations after switching off the $\mu \mathrm{w}$ field (at the delays indicated exemplarily for ${ }^{1} \mathrm{H}$ and ${ }^{13} \mathrm{C}$ in the panel of $100 \mathrm{mM}$ TEMPOL) for a sample containing $I={ }^{1} \mathrm{H}$ and $I={ }^{13} \mathrm{C}$ nuclei. The polarizations are expressed in terms of inverse spin temperatures, $1 / T_{I}$. Traces shown in lighter color indicate the ranges of experimental errors.

69 Zeeman reservoir, introduced to describe energy variations on 70 the order the electronic Zeeman interaction $\nu_{\mathrm{e}}$, and a non71 Zeeman or dipolar reservoir, to describe exchange of energy on 72 the order of the relevant width $\Delta \nu_{\mathrm{e}}$ of the EPR spectrum. (2) 73 Triple-spin flips $S S^{\prime} I$ can establish a contact between the 74 nuclear Zeeman and electron non-Zeeman reservoirs, ${ }^{19}$ since 75 they involve the transfer of energy of the order of the nuclear 76 Zeeman energy $\nu_{I}$ between nuclear and electron spins. (3) The 77 rates of triple-spin flips are fast compared to the rates of other 78 phenomena such as electron or nuclear relaxation rates.

79 Only if these properties are fulfilled can DNP proceed 80 predominantly via TM. In this case, the hyperpolarization 81 process displays two characteristic features:

82 (i) the spin temperatures $T_{I}$ of all nuclear spin species 83 converge to a single common value for long times $t \rightarrow \infty$ 84 in the presence and absence of microwave irradiation. As 85 a result, all nuclei $I$ for which $\nu_{I}<\Delta \nu_{\mathrm{e}}$ are "cooled down" 86 in the same way at all microwave frequencies, in contrast 87 to SE and CE where the microwave frequency for most 88 efficient DNP depends on the nuclear gyromagnetic 89 ratio, $\gamma_{I}$.

90 (ii) A second important consequence of TM is that, if 91 different types of nuclei are present in the sample, heat 92 can flow spontaneously from one nuclear reservoir to 93 another via the non-Zeeman electron reservoir. In other 94 words, if TM is predominant, polarization transfers 95 between different nuclear species are expected to follow 96 the simple laws of heat propagation.

97 In practice, DNP often results not from a single dominant 98 mechanism, but from a combination of different processes such 99 that it may be difficult to separate contributions of different 100 mechanisms. ${ }^{16,20-22}$ A common strategy for the analysis of 101 DNP processes is to simulate experimental results and to assess 102 the underlying mechanism by comparing simulations and 103 experiments. However, this approach is notoriously difficult 104 even for discriminating SE and CE contributions. ${ }^{19}$ When TM comes into play, the superposition of various mechanisms 105 becomes even more difficult to unravel.

106

Therefore, it is currently not clear what DNP mechanisms 107 are active for the frequently used nitroxide-radicals, ${ }^{23}$ in 108 particular at high magnetic fields $B_{0}>6 \mathrm{~T}$ at $T<1.2 \mathrm{~K}$ for 109 D-DNP or $20 \mathrm{~T}$ at $100 \mathrm{~K}$ for MAS-DNP.

110

Here we propose an experimental approach that allows one 111 to determine whether TM is the predominant mechanism in 112 state-of-the-art systems employing nitroxide radicals, based on 113 probing the above-mentioned characteristic features (i) and 114 (ii).

To elucidate the active DNP mechanism and to quantify the 116 flow of polarization between different heat reservoirs via triple 117 spin flips, we performed DNP experiments on spin systems 118 comprising protons $I={ }^{1} \mathrm{H}$ and other nuclei $\left(I^{\prime}={ }^{2} \mathrm{H},{ }^{13} \mathrm{C}\right.$ and 119 $\left.{ }^{31} \mathrm{P}\right)$ in the presence of radicals at variable concentrations. The 120 experiments were carried out in two different ways: (A) we 121 monitored the build-up curves of the polarization $P(I)$ and 122 $P\left(I^{\prime}\right)$ of two different nuclei $I$ and $I^{\prime}$ in the presence of 123 microwave saturation of the EPR spectrum, and subsequently 124 measured their return to thermal equilibrium after interrupting 125 the microwave irradiation; (B) we performed in a systematic 126 way an experiment proposed by Goldman and coauthors for 127 single crystals of $\mathrm{LiF}$ (where $I={ }^{7} \mathrm{Li}$ and $I^{\prime}={ }^{19} \mathrm{~F}$ ), and adapted 128 it to the case of amorphous solids that are used in modern DNP 129 applications. ${ }^{18}$ In this experiment, we polarized two different 130 nuclei (here $I={ }^{1} \mathrm{H}$ and $I^{\prime}={ }^{2} \mathrm{H},{ }^{13} \mathrm{C}$ or ${ }^{31} \mathrm{P}$ ), stopped saturating 131 the EPR transitions, then depolarized one of the nuclei by $\mathrm{rf} 132$ saturation, and observed the subsequent time-dependence of 133 polarizations $P(I)$ and $P\left(I^{\prime}\right)$. This includes a possible 134 spontaneous transfer of polarization between nuclear spin 135 species via the dipolar electron reservoir, which is a clear 136 indication of so-called triple spin flips ( $S S^{\prime} I$ and $S S^{\prime} I^{\prime}$ flips). ${ }_{137}$ These two experiments hence allowed us to monitor to 138 determine whether TM was the dominant mechanism for DNP 139 or not. 
142 Sample Preparation. Sample 1 consisted of glycerol- $\mathrm{d}_{8}, \mathrm{D}_{2} \mathrm{O}$ and $143 \mathrm{H}_{2} \mathrm{O}$ (50:40:10\% in volume). Sample 2 consisted of the same 144 mixture, with additional $1.5 \mathrm{M}$ pyruvate- $1-{ }^{13} \mathrm{C}$. Sample 3 145 consisted of glycerol, glycerol- $\mathrm{d}_{8}, \mathrm{D}_{2} \mathrm{O}$, and $\mathrm{H}_{2} \mathrm{O}$ (with ratios 146 25:25:25:25\% in volume). Sample 4 comprised the same 147 solvent mixture of sample 1 , but contained $0.5 \mathrm{M} \mathrm{K}_{2} \mathrm{HPO}_{4}$ in 148 addition. We used the nitroxide TEMPOL as characterized by a 149 broad inhomogeneous EPR line (ca. $0.5 \mathrm{GHz}$ ). The TEMPOL 150 concentration was varied in the range of $10-100 \mathrm{mM}$.

151 DNP. Our DNP apparatus is described in detail in 152 references. $^{24-26}$ All samples were immersed in liquid helium 153 at $4.2 \mathrm{~K}$ in the cryostat at atmospheric pressure. An ELVA1 154 microwave source coupled to a Virginia Diodes (VDI) 155 frequency doubler provided microwaves $(\mu \mathrm{w})$ at a frequency $156 \nu_{\mu \mathrm{w}}=187.9 \mathrm{GHz}$ for all experiments $\mathbf{A}$ and $\mathbf{B}$. The $\mu \mathrm{w}$-field was 157 modulated over a range of $100 \mathrm{MHz}$ using a sawtooth 158 modulation function with a $1 \mathrm{kHz}$ repetition rate. The 159 microwave frequency profiles were obtained by stepping the 160 central frequency over a range $187.8<\nu_{\mu \mathrm{w}}<188.4 \mathrm{GHz}$ with a $16120 \mathrm{MHz}$ step size and the same modulation bandwidth.

162 We used two-channel NMR probes to detect the polarization 163 of both protons and heteronuclei $I^{\prime}={ }^{2} \mathrm{H},{ }^{13} \mathrm{C}$ or ${ }^{31} \mathrm{P}$ at $B_{0}=6.7$ $164 \mathrm{~T}$. Although TM-DNP leads to higher nuclear polarizations $165 P(I)$ at $1.2 \mathrm{~K}$, the sample temperature was set to $4.2 \mathrm{~K}$ to speed 166 up the polarization build-up and shorten nuclear spin relaxation 167 times, so that the experiments could be run faster. Thus, the 168 inverse sample temperature (and electron spin temperature 169 before microwave saturation) at thermal equilibrium was $1 / T_{0}$ $170=0.25 \mathrm{~K}^{-1}$.

171 In experiment $\mathrm{A}$, the nuclei $I={ }^{1} \mathrm{H}$ and $I^{\prime}={ }^{13} \mathrm{C}$ were 172 polarized through continuous-wave $(\mathrm{cw}) \mu \mathrm{w}$ irradiation to 173 determine the steady-state polarization and the characteristic 174 build-up times of the nuclei. After achieving the steady state, 175 the $\mu \mathrm{w}$ irradiation was interrupted to determine $T_{1}(I)$ and $176 T_{1}\left(I^{\prime}\right)$ decay times. Experiment $\mathbf{B}$ consisted of polarizing ${ }^{1} \mathrm{H}$ 177 nuclei to their steady state by $\mathrm{cw} \mu \mathrm{w}$ irradiation prior to 178 switching off the $\mu \mathrm{w}$ source. The ${ }^{13} \mathrm{C}$ nuclei were then 179 selectively saturated and the time evolution of both ${ }^{1} \mathrm{H}$ and ${ }^{13} \mathrm{C}$ 180 nuclei was subsequently monitored in the absence of $\mu \mathrm{w}$ 181 irradiation. Saturation was achieved by a series of NMR pulses. 182 In all experiments, $1^{\circ}$ detection pulses were used for $I={ }^{1} \mathrm{H}$ 183 and $5^{\circ}$ pulses for $I^{\prime}$ nuclei at intervals of $5 \mathrm{~s}$ to monitor the 184 signal intensity as a function of time.

\section{RESULTS AND DISCUSSION}

186 To elucidate the limits of TM, we systematically varied the 187 experimental conditions for experiments $\mathbf{A}$ and $\mathbf{B}$, to probe the 188 two characteristic signatures (i) and (ii) listed above.

189 (1) Reaching a Common Spin Temperature. Figure 1a displays 190 schematically the flow of spin order in experiment A. Our $\mu \mathrm{w}$ 191 polarizes the non-Zeeman dipolar electronic $\left(e_{\mathrm{NZ}}\right)$ energy levels 192 by cooling down the corresponding heat reservoir. Con193 sequently, triple spin flips tend to equalize the $\mathrm{e}_{\mathrm{NZ}}$ spin 194 temperature and the nuclear spin temperature of both ${ }^{1} \mathrm{H}\left(S S^{\prime} I\right.$ 195 flips) and ${ }^{13} \mathrm{C}$ (SS' $I^{\prime}$ flips) heat reservoirs. After switching off 196 the $\mu \mathrm{w}$ irradiation, both nuclear polarizations $P\left({ }^{1} \mathrm{H}\right)$ and $P\left({ }^{13} \mathrm{C}\right)$ 197 decay and equilibrate at the lattice temperature. Typical DNP 198 build-up curves of these nuclear spin polarization and 199 subsequent decays, obtained for different concentrations of 200 TEMPOL, are shown in Figure 1 b (sample 2).
At all radical concentrations, the nuclei $I={ }^{1} \mathrm{H}$ and $I^{\prime}={ }^{13} \mathrm{C} 201$ have different DNP buildup times and different relaxation 202 times. The polarizations are expressed in units of $1 / T_{I}$, the 203 inverse spin temperature of the corresponding nucleus. The 204 relation between the nuclear spin polarization, $P(I)$, and the 205 inverse spin temperature $\beta_{I}=1 / k_{\mathrm{B}} T_{I}$, is

$$
P(I)=\tanh \left[-\frac{\hbar \omega_{I}}{2 k_{\mathrm{B}} T_{I}}\right]=\tanh \left[-\frac{\beta_{I} \hbar \omega_{I}}{2}\right]
$$

where $\omega_{I}$ is the nuclear Zeeman resonance frequency in the 208 external field. It is readily seen in Figure $1 \mathrm{~b}$ that at TEMPOL 209 concentrations above $50 \mathrm{mM}$, both nuclei converge to a 210 common spin temperature after the initial build-up. In 211 particular, $1 / k_{\mathrm{B}} T_{I}\left({ }^{13} \mathrm{C}\right)$ reaches a maximum when the 212 TEMPOL concentration is $50 \mathrm{mM}$. Most importantly, at 213 lower radical concentrations $(10-40 \mathrm{mM})$, the ${ }^{13} \mathrm{C}$ spin 214 temperature is considerably higher than the proton one, thus 215 indicating that TM is not the dominant mechanism since 216 condition (i) listed above is not fulfilled anymore. ${ }^{12} 217$

The fact that TM no longer dominates at low radical 218 concentrations originates from reduced rates of electron- 219 electron flip-flops as the distance between radical centers 220 becomes larger. On the one hand, when electron spectral 221 diffusion slows down due to decreasing flip-flop probabilities, 222 the electron spins cannot be treated anymore using 223 thermodynamic concepts. Indeed, in this limit, the microwave 224 irradiation will only saturate a narrow band around $\nu_{\mu \mathrm{w}}$ in the 225 EPR spectrum (hole burning), leaving the remaining electron 226 spins at thermal equilibrium with the lattice. On the other hand, 227 the rate of triple-spin flips decreases dramatically and becomes 228 lower than the relaxation rates of the different spin species, 229 leading to breakdown of the thermodynamic description of 230 DNP.

The breakdown of the pertinence of the spin temperature 232 concept at low radical concentrations has been recently 233 predicted by numerical simulations that show a behavior 234 consistent with our observations on ${ }^{13} \mathrm{C}$. The strength of the 235 thermal contact between the $\mathrm{e}_{\mathrm{NZ}}$ reservoir and the nuclear 236 Zeeman reservoir decreases steadily when reducing the radical 237 concentration, down to a point where the thermodynamic 238 description of DNP breaks down and hyperpolarization 239 becomes less effective. ${ }^{27,28}$

(2) Heat Transfer between the Nuclear Zeeman Reservoirs. We 241 can evaluate the presence of triple-spin flips at different radical 242 concentrations by experiment B, schematically depicted in 243 Figure 2a. Triple spin flips offer a mechanism through which $244 \mathrm{f} 2$ the transfer of polarization, i.e., of heat from one nuclear 245 reservoir to another via the $\mathrm{e}_{\mathrm{NZ}}$ reservoir, can take place. Hence, 246 a spontaneous heat flow from a warmer to a colder spin 247 reservoir attests for the presence SSI triple-spin flips. Through 248 our experimental setup, we could observe the transfer of 249 polarization from ${ }^{1} \mathrm{H}$ to ${ }^{13} \mathrm{C}$, i.e., as the ${ }^{1} \mathrm{H}$ reservoir heats up, 250 the ${ }^{13} \mathrm{C}$ reservoir cools down. When the rate of triple spin flips 251 is high, the two nuclear and the $\mathrm{e}_{\mathrm{NZ}}$ reservoirs reach a common 252 spin temperature before they relax to the equilibrium 253 temperature. This is exactly what is observed in Figure 2b 254 between 40 and $100 \mathrm{mM}$, where the ${ }^{13} \mathrm{C}$ polarization of sample 255 2 reaches a maximum when the temperatures of the two 256 nuclear reservoirs become equal (the time traces cross). As 257 such a polarization transfer proceeds via the electronic non- 258 Zeeman reservoir, it can be investigated experimentally by 259 stepping away from ideal TM conditions, e.g., by using lower 260 
a)
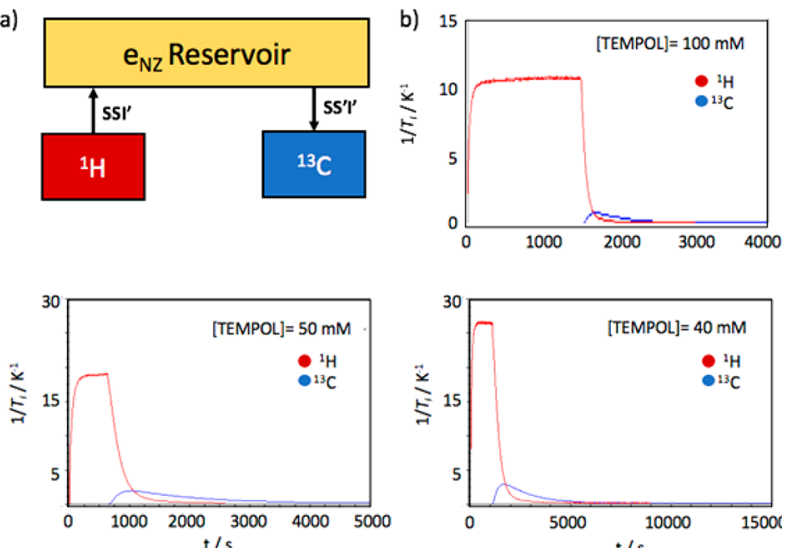

$t / s$

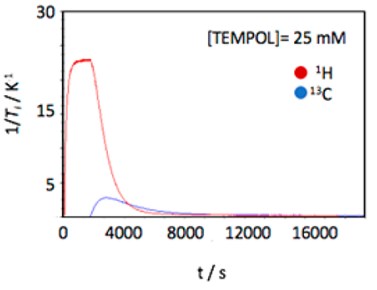

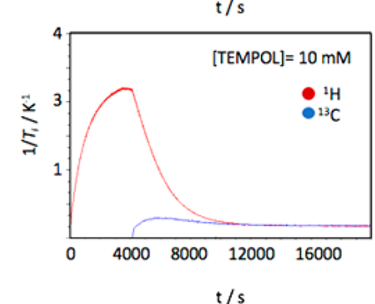

Figure 2. (a) Energy reservoirs involved in experiment $\mathbf{B}$ that monitors the transfer between the polarizations $P\left(I={ }^{1} \mathrm{H}\right)$ and $P\left(I^{\prime}=\right.$ ${ }^{13} \mathrm{C}$ ) after the interruption of the $\mu \mathrm{w}$ irradiation and saturation of either $I$ or $I^{\prime}$ nuclear reservoirs. Via triple spins flips, the polarization flows from ${ }^{1} \mathrm{H}$ to the non-Zeeman dipolar electron reservoir, and is then transferred to ${ }^{13} \mathrm{C}$ spins via $S S^{\prime} I^{\prime}$ triple spin flips. These processes are accompanied by relaxation to the lattice (omitted in the flow diagram). (b) Time evolution of the inverse nuclear spin temperature $1 / T_{I}$. First, a polarization $P\left(I={ }^{1} \mathrm{H}\right)$ is generated by DNP. Subsequently, after the $\mu \mathrm{w}$ irradiation is interrupted, the ${ }^{13} \mathrm{C}$ spins are saturated, and a spontaneous flow of polarization from ${ }^{1} \mathrm{H}$ to ${ }^{13} \mathrm{C}$ reservoirs is observed. Spontaneous transfer of heat from the ${ }^{1} \mathrm{H}$ to ${ }^{13} \mathrm{C}$ reservoir is indicative of triple spin flips $S S^{\prime} I$ and $S S^{\prime} I^{\prime}$.

261 concentrations of radicals or by using radicals with narrow EPR 262 lines.

263 To our surprise, the transfer of polarization between the two 264 nuclear reservoirs is not entirely suppressed, even at a radical 265 concentration as low as $10 \mathrm{mM}$. Thus, even though the 266 signature features of a common spin temperature and an 267 efficient heat transfer are not fulfilled, we observe triple spin 268 flips, indicating a non-TM regime with a reduced rate of such 269 transitions.

Through this, at radical concentrations of $25 \mathrm{mM}$ and below 270 we observe an "anomalous" heat transfer as the maximum 271 inverse spin temperature $1 / k_{\mathrm{B}} T_{I}(\mathrm{C} 13)$ is reached at a time 272 where $1 / k_{\mathrm{B}} T_{I}\left({ }^{1} \mathrm{H}\right)$ is still higher.

273

These data clearly confirm the presence of triple spin flips 274 and transfer of polarization between the Zeeman reservoirs of 275 the ${ }^{1} \mathrm{H}$ and ${ }^{13} \mathrm{C}$ nuclei and the non-Zeeman electronic reservoir, 276 but point to the fact that the rates of triple spin flip transitions 277 involving ${ }^{13} \mathrm{C}$ spins are comparable to or lower than their 278 nuclear relaxation rates, impeding the prevalence of TM as 279 dominant DNP mechanism.

In conclusion, at TEMPOL concentrations of $50 \mathrm{mM}$ or 281 above all characteristic features of TM, as defined above in 282 points (i) and (ii), are observed, while at lower concentrations 283 the nuclear reservoirs do not reach a common spin temper- 284 ature; the heat transfer is inefficient despite the occurrence of 285 triple spin flips.

286

The characteristic build-up times and steady-state spin 287 temperatures at the end of the build-up (experiment A) as 288 well as the transfer times (characteristic cooling times of the 289 ${ }^{13} \mathrm{C}$ reservoir in experiment B) are summarized in Figure 3. $290 \mathrm{f3}$ Note that heat transfer becomes faster with increasing radical 291 concentration (Figure 3c), thereby confirming the dependence 292 of the triple spin flip rate on the TEMPOL concentration. 293

Changing Hyperpolarized Nuclei. To shed more light on the 294 mechanistic details at intermediate TEMPOL concentrations, 295 where features (i) (similar spin temperatures) and (ii) 296 (characteristic heat transfer) of TM are not fulfilled, but triple 297 spin flips are still observed via experiment B, we measured the 298 dependence of the spin polarization $P\left(I^{\prime}\right)$ of the four nuclei $I^{\prime}=299$ ${ }^{1} \mathrm{H},{ }^{13} \mathrm{C},{ }^{2} \mathrm{H}$ and ${ }^{31} \mathrm{P}$ on the microwave irradiation frequency $\nu_{\mu \mathrm{w}} 300$ at $25 \mathrm{mM}$ TEMPOL. The profiles are shown in Figure 4. When $301 \mathrm{f} 4$ $\mathrm{TM}$ is dominant, the widths of the $\mu \mathrm{w}$-profiles would primarily 302 depend the properties of the EPR spectrum of TEMPOL. ${ }^{12,19} 303$ However, it is readily seen that the profiles for ${ }^{1} \mathrm{H}$ and ${ }^{2} \mathrm{H}$ are 304 similar, while they differ significantly from those of ${ }^{13} \mathrm{C}$ and ${ }^{31} \mathrm{P}, 305$ which show slight differences between each other. In cases of 306 $\mathrm{CE}$ or $\mathrm{SE}$, one would expect a frequency difference of $\nu_{I}$ or $2 \nu_{I}, 307$ respectively, between the maxima and minima of the microwave 308 profiles and thus a strong dependence on the nuclear 309 gyromagnetic ratio.

Since none of these features is observed for any nuclei, we 311 assume a mixture of different mechanisms for the low radical 312 concentration regime. For ${ }^{1} \mathrm{H}$ and ${ }^{2} \mathrm{H}$ we observe profiles where 313 the extrema are separated by ca. $440 \mathrm{MHz}$ (more than the 314
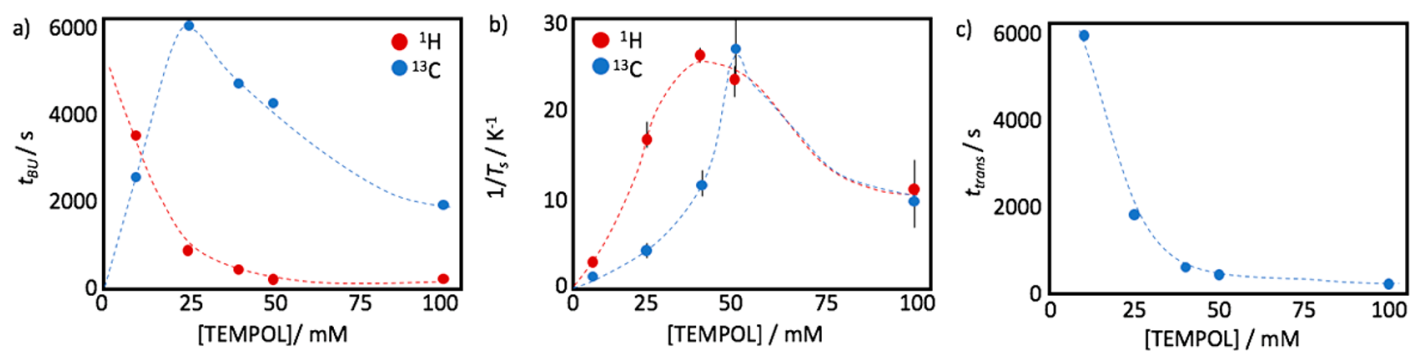

Figure 3. (a) Characteristic build-up times, $t_{\mathrm{BU}}$ for $P\left({ }^{1} \mathrm{H}\right)$ and $P\left({ }^{13} \mathrm{C}\right)$ polarizations at different TEMPOL concentrations, obtained by fitting experimental build-up curves to monoexponential functions. At low radical concentrations, the buildup of $P\left({ }^{13} \mathrm{C}\right)$ is faster than the buildup of $P\left({ }^{1} \mathrm{H}\right)$, indicating a change of the dominant DNP regime (see text for details). (b) Dependence of the inverse steady-state spin temperatures $1 / T_{\mathrm{I}}\left({ }^{1} \mathrm{H}\right)$ and $1 / T_{\mathrm{I}}\left({ }^{13} \mathrm{C}\right)$ as a function of the radical concentration. At a TEMPOL concentration of $50 \mathrm{mM}$, these inverse spin temperatures are similar within experimental error, indicating that TM is predominant, while below $40 \mathrm{mM}$ this is no longer the case. (c) Characteristic polarization transfer times from $P\left({ }^{1} \mathrm{H}\right)$ to $P\left({ }^{13} \mathrm{C}\right)$ obtained by fitting the first part of the ${ }^{13} \mathrm{C}$ time dependence in experiment $\mathbf{B}$ to monoexponential build-up functions. The dashed lines are to guide the eye. 


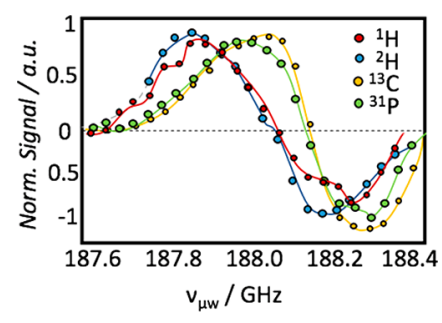

Figure 4. Dependence of nuclear signal intensities on the microwave frequency (" $\mu \mathrm{w}$ profiles") detected for ${ }^{1} \mathrm{H}$ (sample 1), on ${ }^{13} \mathrm{C}$ (sample 2 ), on ${ }^{2} \mathrm{H}$ (sample 3) and on ${ }^{31} \mathrm{P}$ (sample 4). In all cases, the frequency differences between the maxima and minima of the $\mu \mathrm{w}$ profiles do not correspond to multiples of $\omega_{I}$, indicating significant contributions of TM; however, slight differences between the profiles indicate admixtures of SE and CE.

315 proton Larmor frequency, which is $\nu_{I}=285.3 \mathrm{MHz}$ at $6.7 \mathrm{~T}$ ), 316 and for ${ }^{13} \mathrm{C}$ and ${ }^{31} \mathrm{P}$ the extrema are separated by ca. $300 \mathrm{MHz}$. 317 Vega, Han, and co-workers ${ }^{13,14,16}$ have shown, based on 318 simulations of experimental data under similar conditions, 319 that at $25 \mathrm{mM}$ TEMPOL for ${ }^{1} \mathrm{H}$, a combination of CE and DSE 320 can reproduce the experimental observations, in agreement 321 with our observation that the ${ }^{1} \mathrm{H}$ and ${ }^{13} \mathrm{C}$ spin reservoirs do not 322 reach a common spin temperature at this radical concentration. 323 This indicates that the description of the DNP process as 324 "pure" TM breaks down. We observe a similar behavior for ${ }^{2} \mathrm{H}$, ${ }_{325}{ }^{13} \mathrm{C}$ and ${ }^{31} \mathrm{P}$. Note that the difference between the $\mu \mathrm{w}$ profiles 326 characteristic for TM and CE is very subtle (the $\mu \mathrm{w}$ profiles for ${ }_{327}{ }^{1} \mathrm{H}$ can be reproduced both with TM-based as well as CE-based 328 models). ${ }^{13,24}$

329 Interestingly, we observe for all these nuclei triple spin flips 330 via experiment $\mathbf{B}$ at $25 \mathrm{mM}$ TEMPOL (see Figure 5).

331 For pairs of $I={ }^{1} \mathrm{H}$ with $I^{\prime}={ }^{13} \mathrm{C},{ }^{2} \mathrm{H}$ or ${ }^{31} \mathrm{P}$, one finds a 332 spontaneous heat transfer via the $\mathrm{e}_{\mathrm{NZ}}$ reservoir (see Figure $5 \mathrm{a}, \mathrm{b}$ 333 for the spin temperature as a function of time for $I^{\prime}={ }^{2} \mathrm{H}$ and $334{ }^{31} \mathrm{P}$ ). This clearly shows that the presence of triple spin flips is 335 not a sufficient condition for TM, though a necessary one, if we 336 assume TM as strictly defined by the signature features listed 337 above.

338 Additionally, in experiment $\mathbf{B}$, the heat transfer between ${ }^{13} \mathrm{C}$ 339 and ${ }^{1} \mathrm{H}$ via the $\mathrm{e}_{\mathrm{NZ}}$ reservoir with $50 \mathrm{mM}$ TEMPOL works both 340 ways (see Figure 5c), which confirms the consistency of our 341 interpretation. In contrast, radicals with narrow EPR lines like $34250 \mathrm{mM}$ BDPA do not lead to any observable heat transfer in 343 experiment B. This excludes direct cross-talk between the 344 nuclear reservoirs (data not shown).

\section{CONCLUSIONS}

345

Combining experiments A and B yields a strategy for the 346 determination of TM contributions to DNP, corresponding to 347 the limiting case where the spin temperatures of all spin 348 reservoirs tend to a common value $T_{I}$ for $t \rightarrow \infty$ due to triple 349 spin flips and the spontaneous heat transfer between different 350 reservoirs. This strategy allows one to assess the presence of the 351 TM regime, understood as a situation where a common nuclear 352 spin temperature emerges from a spontaneous heat flow, 353 through triple spin flip transitions. These two necessary 354 conditions must both be fulfilled to establish TM, while one 355 of them alone is insufficient. We want to stress that the sole 356 occurrence of triple spin flips, or of spontaneous heat transfer, 357 is not indicative of strict TM, if the spin temperatures of the 358 different nuclei in the system do not converge. It can be clearly 359 stated that TM is the dominant mechanism at high TEMPOL 360 concentrations $(\geq 50 \mathrm{mM})$ at $4.2 \mathrm{~K}$ and $6.7 \mathrm{~T}$, i.e., for 361 experimental conditions frequently used in modern DNP 362 experiments, while for lower TEMPOL concentrations $(\leq 40363$ $\mathrm{mM}$ ) the spin temperatures of the different reservoirs can be 364 different. The latter fact, in combination with the observation of 365 spontaneous heat transfer, indicates the presence of $S S^{\prime} I$ flips 366 outside of the TM regime, which corresponds to a mixture of 367 different mechanisms. At TEMPOL concentrations of $10 \mathrm{mM}, 368$ where we observe only very weak heat transfer (experiment B 369 indicates the breakdown of $S S^{\prime} I$ transitions), the DSE is the 370 most likely mechanism, as pointed out by $\mathrm{Han}$ and co- 371 workers. $^{13}$

From theoretical considerations, we expect the TM regime to 373 establish at even lower radical concentrations if the sample 374 temperature or the external magnetic field are decreased. 375 Considering that processes, which involve a heat flow, need to 376 compete with electronic $T_{1}$ relaxation, it appears intuitive that 377 conditions that prolong relaxation favor thermal mixing. $\quad 378$

From the experimental point of view, it is important to note 379 that the lowest ${ }^{13} \mathrm{C}$ spin temperature can be reached at 380 TEMPOL concentrations of $50 \mathrm{mM}$ due to low ${ }^{1} \mathrm{H}$ spin 381 temperatures and the dominance of TM. Yet, even lower ${ }^{1} \mathrm{H} 382$ temperatures can be reached at a lower TEMPOL concen- 383 tration of $40 \mathrm{mM}$ because favorable saturation factors. ${ }^{29,30}$ This 384 information is crucial for users who cannot rely on cross- 385 polarization techniques. ${ }^{26}$ In this situation TM-DNP can be a 386 remedy, since it not only allows one to hyperpolarize protons, 387 but various other nuclei by exploiting additional pathways for 388 efficient polarization transfer.

AUTHOR INFORMATION 390

Corresponding Authors 391

*E-mail: kurzbach@ens.fr (D.K.).
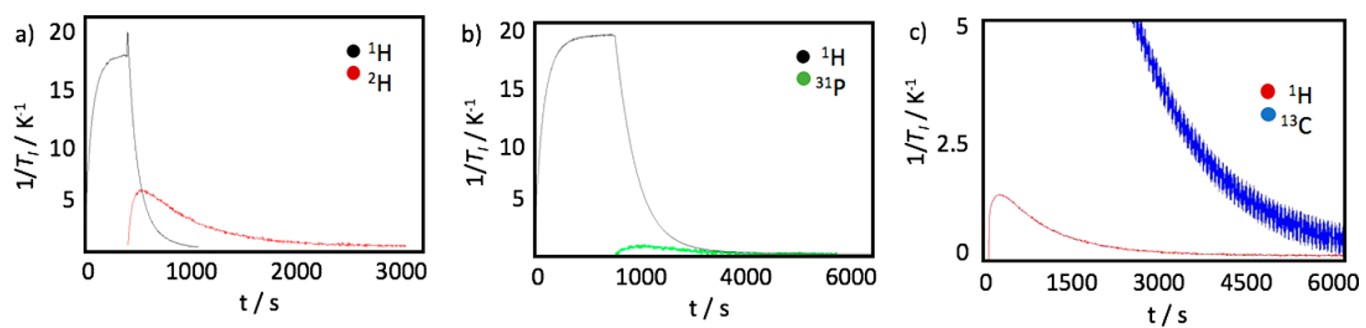

Figure 5. Experiments B for (a) sample 2 and (b) sample 4. For both ${ }^{2} \mathrm{H}$ and ${ }^{31} \mathrm{P}$ a spontaneous polarization transfer from ${ }^{1} \mathrm{H}$ can be observed with $25 \mathrm{mM}$ TEMPOL. (c) ${ }^{13} \mathrm{C}$ to ${ }^{1} \mathrm{H}$ polarization transfer in experiment $\mathbf{B}$ for sample 2 containing $50 \mathrm{mM}$ TEMPOL after polarizing ${ }^{13} \mathrm{C}$ and saturating ${ }^{1} \mathrm{H}$. Evidently, triple spin flips can transfer heat in both directions. 
393 *E-mail: ivanov@tomo.nsc.ru (K.L.I.).

\section{ORCID 다}

395 Dennis Kurzbach: 0000-0001-6455-2136

396 Notes

397 The authors declare no competing financial interest.

\section{ACKNOWLEDGMENTS}

399 The authors thank Dr. Maurice Goldman for helpful 400 discussions and Bruker BioSpin for providing a prototype of 401 the DNP equipment. This work was supported by the French 402 CNRS and the European Research Council contract "dilute 403 para-water". K.L.I. acknowledges ENS (Paris) for a guest 404 professorship and the Russian Foundation for Basic Research 405 (Grant No. 17-03-00932). A.R. acknowledges ANR-16-CE30406 0023-01 (THERMOLOC).

\section{REFERENCES}

408 (1) Valla, M.; Rossini, A. J.; Caillot, M.; Chizallet, C.; Raybaud, P.; 409 Digne, M.; Chaumonnot, A.; Lesage, A.; Emsley, L.; van Bokhoven, J. 410 A.; Coperet, C. Atomic Description of the Interface between Silica and 411 Alumina in Aluminosilicates through Dynamic Nuclear Polarization 412 Surface-Enhanced NMR Spectroscopy and First-Principles Calcu413 lations. J. Am. Chem. Soc. 2015, 137, 10710-10719.

414 (2) Lesage, A.; Lelli, M.; Gajan, D.; Caporini, M. A.; Vitzthum, V.; 415 Mieville, P.; Alauzun, J.; Roussey, A.; Thieuleux, C.; Mehdi, A.; 416 Bodenhausen, G.; Coperet, C.; Emsley, L. Surface enhanced NMR 417 spectroscopy by dynamic nuclear polarization. J. Am. Chem. Soc. 2010, 418 132, 15459-61.

419 (3) Rosay, M.; Tometich, L.; Pawsey, S.; Bader, R.; Schauwecker, R.; 420 Blank, M.; Borchard, P. M.; Cauffman, S. R; Felch, K. L.; Weber, R. 421 T.; Temkin, R. J.; Griffin, R. G.; Maas, W. E. Solid-state dynamic 422 nuclear polarization at $263 \mathrm{GHz}$ : spectrometer design and 423 experimental results. Phys. Chem. Chem. Phys. 2010, 12, 5850-60.

424 (4) Potapov, A.; Thurber, K. R.; Yau, W. M.; Tycko, R. Dynamic 425 nuclear polarization-enhanced (1) H-(1)(3)C double resonance NMR 426 in static samples below $20 \mathrm{~K}$. J. Magn. Reson. 2012, 221, 32-40.

427 (5) Dumez, J. N.; Milani, J.; Vuichoud, B.; Bornet, A.; Lalande428 Martin, J.; Tea, I.; Yon, M.; Maucourt, M.; Deborde, C.; Moing, A.; 429 Frydman, L.; Bodenhausen, G.; Jannin, S.; Giraudeau, P. Hyper430 polarized NMR of plant and cancer cell extracts at natural abundance. 431 Analyst 2015, 140, 5860-5863.

432 (6) Miclet, E.; Abergel, D.; Bornet, A.; Milani, J.; Jannin, S.; 433 Bodenhausen, G. Toward Quantitative Measurements of Enzyme 434 Kinetics by Dissolution Dynamic Nuclear Polarization. J. Phys. Chem. 435 Lett. 2014, 5, 3290-5.

436 (7) Wilson, D. M.; Kurhanewicz, J. Hyperpolarized C-13 MR for 437 Molecular Imaging of Prostate Cancer. J. Nucl. Med. 2014, 55, 15674381572.

439 (8) Tayler, M. C.; Marco-Rius, I.; Kettunen, M. I.; Brindle, K. M.; 440 Levitt, M. H.; Pileio, G. Direct enhancement of nuclear singlet order 441 by dynamic nuclear polarization. J. Am. Chem. Soc. 2012, 134, 766844271 .

443 (9) Ardenkjaer-Larsen, J. H.; Fridlund, B.; Gram, A.; Hansson, G.; 444 Hansson, L.; Lerche, M. H.; Servin, R.; Thaning, M.; Golman, K. 445 Increase in signal-to-noise ratio of $>10,000$ times in liquid-state NMR. 446 Proc. Natl. Acad. Sci. U. S. A. 2003, 100, 10158-63.

447 (10) Kurzbach, D.; Canet, E.; Flamm, A. G.; Jhajharia, A.; Weber, E. 448 M.; Konrat, R.; Bodenhausen, G. Investigation of Intrinsically 449 Disordered Proteins through Exchange with Hyperpolarized Water. 450 Angew. Chem., Int. Ed. 2017, 56, 389-392.

451 (11) Chappuis, Q.; Milani, J.; Vuichoud, B.; Bornet, A.; Gossert, A. 452 D.; Bodenhausen, G.; Jannin, S. Hyperpolarized Water to Study 453 Protein-Ligand Interactions. J. Phys. Chem. Lett. 2015, 6, 1674-1678. 454 (12) Abragam, A.; Goldman, M. Principles of Dynamic Nuclear455 Polarization. Rep. Prog. Phys. 1978, 41, 395-467.

456 (13) Leavesley, A.; Shimon, D.; Siaw, T. A.; Feintuch, A.; Goldfarb, 457 D.; Vega, S.; Kaminker, I.; Han, S. Effect of electron spectral diffusion on static dynamic nuclear polarization at 7 T. Phys. Chem. Chem. Phys. 458 2017, 19, 3596-3605.

(14) Shimon, D.; Hovav, Y.; Feintuch, A.; Goldfarb, D.; Vega, S. 460 Dynamic nuclear polarization in the solid state: a transition between 461 the cross effect and the solid effect. Phys. Chem. Chem. Phys. 2012, 14, 462 $5729-5743$.

(15) Wenckebach, W. T. Dynamic nuclear polarization via thermal 464 mixing: Beyond the high temperature approximation. J. Magn. Reson. 465 2017, 277, 68-78.

(16) Hovav, Y.; Feintuch, A.; Vega, S. Theoretical aspects of dynamic 467 nuclear polarization in the solid state - spin temperature and thermal 468 mixing. Phys. Chem. Chem. Phys. 2013, 15, 188-203.

469

(17) Serra, S. C.; Rosso, A.; Tedoldi, F. Electron and nuclear spin 470 dynamics in the thermal mixing model of dynamic nuclear 471 polarization. Phys. Chem. Chem. Phys. 2012, 14, 13299-13308. 472

(18) Cox, S. F. J.; Bouffard, V.; Goldman, M. The coupling of two 473 nuclear Zeeman reservoirs by the electronic spin-spin reservoir. J. Phys. 474 C: Solid State Phys. 1973, 6, L100-L103.

(19) Wenckebach, T. Essentials of Dynamic Nuclear Polarization; 476 Spindrift Publications: The Netherlands, 2016.

(20) Hovav, Y.; Levinkron, O.; Feintuch, A.; Vega, S. Theoretical 478 Aspects of Dynamic Nuclear Polarization in the Solid State: The 479 Influence of High Radical Concentrations on the Solid Effect and 480 Cross Effect Mechanisms. Appl. Magn. Reson. 2012, 43, 21-41. 481

(21) Hovav, Y.; Feintuch, A.; Vega, S. Theoretical aspects of dynamic 482 nuclear polarization in the solid state - The cross effect. J. Magn. Reson. 483 2012, 214, 29-41.

(22) Hovav, Y.; Feintuch, A.; Vega, S. Theoretical aspects of dynamic 485 nuclear polarization in the solid state - The solid effect. J. Magn. Reson. 486 2010, 207, 176-189.

487

(23) Sauvee, C.; Casano, G.; Abel, S.; Rockenbauer, A.; 488 Akhmetzyanov, D.; Karoui, H.; Siri, D.; Aussenac, F.; Maas, W.; 489 Weber, R. T.; Prisner, T.; Rosay, M.; Tordo, P.; Ouari, O. Tailoring of 490 Polarizing Agents in the bTurea Series for Cross-Effect Dynamic 491 Nuclear Polarization in Aqueous Media. Chem. - Eur. J. 2016, 22, 492 5598-606.

(24) Weber, E. M. M.; Vezin, H.; Kempf, J. G.; Bodenhausen, G.; 494 Abergel, D.; Kurzbach, D. Anisotropic longitudinal electronic 495 relaxation affects DNP at cryogenic temperatures. Phys. Chem. Chem. 496 Phys. 2017, 19, 16087-16094.

(25) Kurzbach, D.; Weber, E. M. M.; Jhajharia, A.; Cousin, S. F.; 498 Sadet, A.; Marhabaie, S.; Canet, E.; Birlirakis, N.; Milani, J.; Jannin, S.; 499 Eshchenko, D.; Hassan, A.; Melzi, R.; Luetolf, S.; Sacher, M.; Rossire, 500 M.; Kempf, J.; Lohman, J. A. B.; Weller, M.; Bodenhausen, G.; Abergel, 501 D. Dissolution Dynamic Nuclear Polarization of Deuterated Molecules 502 Enhanced by Cross-Polarization. J. Chem. Phys. 2016, 145, 194203.503

(26) Jannin, S.; Bornet, A.; Colombo, S.; Bodenhausen, G. Low- 504 temperature cross polarization in view of enhancing dissolution 505 Dynamic Nuclear Polarization in NMR. Chem. Phys. Lett. 2011, 517, 506 234-236.

(27) De Luca, A.; Rosso, A. Dynamic nuclear polarization and the 508 paradox of Quantum Thermalization. Phys. Rev. Lett. 2015, 115, 509 080401-1-080401-5.

(28) De Luca, A.; Rodriguez-Arias, I.; Mueller, M.; Rosso, A. 511 Thermalization and many-body localization in systems under dynamic 512 nuclear polarization. Phys. Rev. B: Condens. Matter Mater. Phys. 2016, 513 94, 014203-1-014203-15.

514

(29) Siaw, T. A.; Fehr, M.; Lund, A.; Latimer, A.; Walker, S. A.; 515 Edwards, D. T.; Han, S. I. Effect of electron spin dynamics on solid- 516 state dynamic nuclear polarization performance. Phys. Chem. Chem. 517 Phys. 2014, 16, 18694-18706.

(30) Serra, S. C.; Rosso, A.; Tedoldi, F. On the role of electron- 519 nucleus contact and microwave saturation in thermal mixing DNP. 520 Phys. Chem. Chem. Phys. 2013, 15, 8416-8428. 